\title{
Effect of bivalent human papillomavirus vaccination on pregnancy outcomes: long term observational follow-up in the Costa Rica HPV Vaccine Trial
}

\author{
Orestis A Panagiotou, ${ }^{1}$ Brian L Befano, ${ }^{2}$ Paula Gonzalez,, 3,4 Ana Cecilia Rodríguez, ${ }^{3}$ \\ Rolando Herrero, ${ }^{4}$ John T Schiller, ${ }^{5}$ Aimée R Kreimer, ${ }^{1}$ Mark Schiffman, ${ }^{1}$ Allan Hildesheim, ${ }^{1}$ \\ Allen J Wilcox, ${ }^{6}$ Sholom Wacholder, ${ }^{1}$ on behalf of the Costa Rica HPV Vaccine Trial (CVT) Group \\ (see end of manuscript for full list of investigators)
}

\section{ABSTRACT}

OBJECTIVE

To examine the effect of the bivalent human papillomavirus (HPV) vaccine on miscarriage. DESIGN

Observational long term follow-up of a randomized, double blinded trial combined with an independent unvaccinated population based cohort.

SETTING

Single center study in Costa Rica.

PARTICIPANTS

7466 women in the trial and 2836 women in the unvaccinated cohort enrolled at the end of the randomized trial and in parallel with the observational trial component.

\section{INTERVENTION}

Women in the trial were assigned to receive three doses of bivalent HPV vaccine $(n=3727)$ or the control hepatitis A vaccine $(n=3739)$. Crossover bivalent HPV vaccination occurred in the hepatitis $A$ vaccine arm at the end of the trial. Women in the unvaccinated cohort received ( $n=2836)$ no vaccination.

\section{MAIN OUTCOME MEASURE}

Risk of miscarriage, defined by the US Centers for Disease Control and Prevention as fetal loss within 20 weeks of gestation, in pregnancies exposed to bivalent HPV vaccination in less than 90 days and any time from vaccination compared with pregnancies exposed to hepatitis $A$ vaccine and pregnancies in the unvaccinated cohort.

RESULTS

Of 3394 pregnancies conceived at any time since bivalent HPV vaccination, 381 pregnancies were

\section{WHAT IS ALREADY KNOWN ON THIS TOPIC}

Bivalent vaccination against human papillomavirus types 16 and 18 is part of the recommended immunization schedule in many countries

Previous studies could not rule out on effect on miscarriage of the bivalent vaccine, especially for pregnancies conceived less than 90 days after vaccination

\section{WHAT THIS STUDY ADDS}

There is no increased risk of miscarriage for pregnancies conceived less than 90 days or any time after vaccination

An observed increased risk for miscarriages in gestation weeks 13-20 may be a random finding as a result of thorough examination of several possible scenarios, but it should be further explored in existing and future studies conceived less than 90 days from vaccination. Unexposed pregnancies comprised 2507 pregnancies conceived after hepatitis A vaccination and 720 conceived in the unvaccinated cohort. Miscarriages occurred in 451 (13.3\%) of all exposed pregnancies, in 50 (13.1\%) of the pregnancies conceived less than 90 days from bivalent HPV vaccination, and in 414 (12.8\%) of the unexposed pregnancies, of which 316 $(12.6 \%)$ were in the hepatitis A vaccine group and 98 $(13.6 \%)$ in the unvaccinated cohort. The relative risk of miscarriage for pregnancies conceived less than 90 days from vaccination compared with all unexposed pregnancies was 1.02 (95\% confidence interval 0.78 to 1.34 , one sided $P=0.436$ ) in unadjusted analyses. Results were similar after adjusting for age at vaccination (relative risk 1.15 , one sided $P=0.17$ ), age at conception (1.03, $\mathrm{P}=0.422$ ), and calendar year (1.06, $\mathrm{P}=0.358$ ), and in stratified analyses. Among pregnancies conceived at any time from bivalent HPV vaccination, exposure was not associated with an increased risk of miscarriage overall or in subgroups, except for miscarriages at weeks 13-20 of gestation (relative risk $1.35,95 \%$ confidence interval 1.02 to 1.77 , one sided $\mathrm{P}=0.017$ ).

\section{CONCLUSIONS}

There is no evidence that bivalent HPV vaccination affects the risk of miscarriage for pregnancies conceived less than 90 days from vaccination. The increased risk estimate for miscarriages in a subgroup of pregnancies conceived any time after vaccination may be an artifact of a thorough set of sensitivity analyses, but since a genuine association cannot totally be ruled out, this signal should nevertheless be explored further in existing and future studies.

TRIAL REGISTRATION

Clinicaltrials.gov NCT00128661 and NCT01086709.

\section{Introduction}

Prophylactic vaccination against the human papillomavirus (HPV) is now recommended as part of the vaccination schedules in many countries, with millions of women worldwide receiving either the bivalent vaccine against HPV types 16 and 18 or the quadrivalent vaccine against types $6,11,16$, and $18 .^{1-3}$ No major safety concerns for either vaccine have been documented in prelicensure or post-licensure studies to date, ${ }^{45}$ as is also reflected in recent reports by the US Agency for Health Research and Quality, ${ }^{6}$ the Institute of Medicine, ${ }^{7}$ the Advisory Committee on Immunization 
Practices of the Centers for Disease Control and Prevention, ${ }^{2}$ and the World Health Organization. ${ }^{8}$

Nevertheless, in 2010 the data safety monitoring board of the randomized bivalent HPV vaccination Costa Rica HPV Vaccine Trial (CVT) raised concerns about the effect of vaccination on the risk of miscarriage in the parallel PApilloma TRIal against Cancer In young Adults (PATRICIA). In a pooled analysis of the two trials, Wacholder and colleagues found no evidence supporting an increased risk of miscarriage for pregnancies conceived at least 90 days after vaccination using the bivalent HPV vaccine. ${ }^{9}$ Their observation of an imbalance in miscarriage rates for pregnancies conceived within 90 days from vaccination in the bivalent HPV vaccination arm compared with the control arm (13.7\% $v 9.2 \%$, one sided $\mathrm{P}=0.033$ ) did not allow investigators to "completely rule out the possibility of an increased risk among pregnancies conceived within three months of vaccination." Although the primary target of HPV vaccination are girls aged 11-13 years (depending on the country), national immunization schedules in many countries recommend vaccination for females until the age of 25 or 26 years. ${ }^{210}$ Hence substantial numbers of women of reproductive age around the world are anticipated to be exposed to vaccination with the bivalent HPV vaccine. Pregnancy related adverse effects of any magnitude in these women are of primary importance from both a clinical practice and a public health point of view as they can affect personal decisions about vaccination, thus mitigating the benefits of vaccinationthat is, the prevention of clinically relevant endpoints of precancerous lesions and cervical cancer. ${ }^{112}$

Currently, evidence on a causal effect of HPV vaccination on miscarriage warrants further investigation. ${ }^{6}$ To address potential concerns for the effect of vaccination with the bivalent HPV vaccine on miscarriage, we analyzed pregnancy outcomes in the trial phase and the post-trial follow-up of the Costa Rica HPV Vaccine Trial using a larger sample size with longer follow-up periods than in our previous interim analysis. ${ }^{9}$ We were able to include pregnancies from the randomized, blinded phase of the trial, pregnancies in the post-trial phase after women in the control arm were offered crossover vaccination with bivalent HPV vaccine thus permitting additional observations of miscarriage risk after vaccination, and pregnancies in an unvaccinated cohort.

\section{Methods}

Study population

The design of the Costa Rica HPV Vaccine Trial and the eligibility criteria have been described in detail elsewhere. ${ }^{13-15}$ In the current analysis we included data from the randomized, blinded phase of the trial and the posttrial long term follow-up study, in which participants were no longer randomized. In brief, the trial is a double blind randomized controlled trial that evaluated the efficacy of the bivalent HPV vaccine.

Women aged 18-25 years in Guanacaste, Costa Rica, who met the eligibility criteria (see supplementary file), were enrolled from June 2004 to December 2005. They were randomly assigned to either the experimental bivalent HPV L1 virus-like particle ASO4 vaccine ( $n=3727$ women) or the control hepatitis A vaccine ( $n=3739$ women). Women in both arms were scheduled to receive three doses of the respective intervention (one dose at baseline, one month, and six months) and were followed annually for four years. In accordance with the consent form, participants were unblinded to their vaccine status at the end of the blinded phase of the trial and offered crossover vaccination. This included a hepatitis B vaccine and the study vaccine (bivalent HPV or hepatitis A) that they did not receive at enrolment into the trial (see supplementary file).

After completion of the four year follow-up period of the trial phase, a non-randomized, observational posttrial long term follow-up study was implemented, with the goal of extending follow-up for an additional six years (see supplementary file). ${ }^{15}$

In addition, we enrolled an unvaccinated cohort ( $n=2836$ ) to allow continued evaluation of efficacy and safety of the vaccine despite the loss of the original control arm owing to crossover. Enrolment in the unvaccinated cohort was contemporaneous with the final study visit of the randomized, blinded phase. Women were frequency matched by birth year and geographic area to the originally randomized women and were sampled randomly from a local census $(n=22240)$. Women's characteristics and predicted future risk of acquisition of cervical HPV in years 4-10 were similar to those of women in the hepatitis A vaccine arm. ${ }^{15}$

\section{Endpoints}

The primary endpoint was miscarriage, defined as pregnancy loss within 20 weeks of gestation, estimated based on the last reported menstrual period. We used 20 weeks as the upper threshold to characterize an in utero pregnancy loss as miscarriage following the definitions set by the National Center for Health Statistics of the US Centers for Disease Control and Prevention. ${ }^{91617}$ We excluded molar and ectopic pregnancies, which are rare and likely to have different causes from those of most miscarriages. We also did not consider pregnancy terminations (induced abortions), as these are not legal in Costa Rica. We also examined two secondary endpoints: total number of conceived pregnancies regardless of their resolution status, and live births.

The unit of analysis was pregnancies. We handled each of two or more pregnancies from the same woman as an independent event. For the endpoint of miscarriage, we included all resolved pregnancies by the 20th week of gestation occurring in the two arms during the first phase of the trial and all pregnancies that occurred in the long term follow-up study from date of exit from the first phase through the data freeze date of 20 December 2013 (year 4 of the long term follow-up study). For the endpoint of live births, we included all resolved pregnancies where outcome was other than loss; whereas for the endpoint of total number of conceived pregnancies, we included all conceptions regardless of their subsequent resolution. We calculated the time between conception and vaccination as the number of days between the estimated conception of a pregnancy 
and the nearest vaccination that was administered before the end of the pregnancy. Conception date was estimated as 14 days after the last menstrual period. We excluded pregnancies for which the last menstrual period was missing.

\section{Pregnancy determination}

Participants had to be using some form of contraception, including abstinence, at least one month before the application of the vaccine and be willing to use it until two months after the last dose. During the first phase of the trial and crossover vaccination, women who either were known to be pregnant or had a positive pregnancy test at the time of enrolment were deferred until three months post partum. If pregnancy was reported or detected at another vaccination visit the mother was not eligible to receive further vaccines. Similarly, a woman was not eligible for subsequent vaccinations after a reported miscarriage.

In the blinded phase of the trial, a $\beta$ human chorionic gonadotropin pregnancy test was performed in all women before vaccination with either bivalent HPV or hepatitis A. At crossover, a pregnancy test was performed for women accepting bivalent HPV vaccination. No pregnancy test was performed for the crossover hepatitis A or bivalent HPV vaccination, since there is no evidence that these vaccines affect pregnancy outcomes. No such test is required in routine clinical practice. ${ }^{6}$

\section{Comparison groups for exposed and unexposed pregnancies}

Exposed and unexposed pregnancies for the miscarriage endpoint are described in detail in the supplementary file. Exposed to bivalent HPV vaccine were pregnancies in women initially randomized to bivalent HPV vaccine in the main trial with conception dates either in that trial or in the long term follow-up study, and pregnancies exposed to bivalent HPV vaccine as a result of the crossover. Unexposed were all pregnancies in women initially randomized to the hepatitis A virus arm during the main trial, pregnancies not exposed to bivalent HPV crossover vaccination, and pregnancies in the unvaccinated cohort.

For the endpoints of miscarriage and live births, in the main analysis we focused on pregnancies exposed to bivalent HPV vaccine in the time window with the highest prior probability of an effect-that is, pregnancies with an estimated conception date up to 12 weeks ( $<90$ days) before or after vaccination. Hence we compared the miscarriage rates among pregnancies conceived from 89 days before to 89 days after the nearest bivalent HPV vaccination to the miscarriage rates among all unexposed pregnancies. In this analysis we excluded pregnancies with an estimated date of conception of 90 days or more from vaccination. For the unexposed groups we assumed that the miscarriage rate for pregnancies after hepatitis A vaccination is constant and independent of (that is, unrelated to) the time of vaccination as in the unvaccinated cohort, since we have no prior evidence that hepatitis A vaccine, which has been given to millions of women around the world, might have any effect (beneficial or harmful) on miscarriage. ${ }^{18-20}$ To fully explore a more extended possible effect of bivalent HPV vaccine on miscarriage risk, we examined the miscarriage risk for pregnancies conceived at any time point relative to bivalent HPV vaccination (that is, without restricting to those in the 89 day window before or after vaccination) compared with all unexposed pregnancies.

For the endpoint of total number of conceived pregnancies, the analyses of pregnancy rates pertain to the number of conceptions regardless of their subsequent resolution. Vaccination with bivalent HPV could affect conception only if it precedes conception. Thus, pregnancies with an estimated conception date after vaccination are considered exposed, whereas pregnancies with an estimated conception date before vaccination are considered unexposed. Women receiving hepatitis A vaccine and those in the unvaccinated cohort are also considered unexposed.

\section{Subgroup and stratified analyses}

In subgroup analyses we restricted to pregnancies conceived less than 90 days before or after vaccination in both exposure groups-that is, less than 90 days before or after bivalent HPV vaccination and less than 90 days before or after hepatitis A vaccination. This analysis excluded the unvaccinated cohort, for which no vaccination had been performed and thus no time window could be specified. We also restricted the endpoint of miscarriage according to gestational age when the miscarriage occurred (0-6, 7-12, and 13-20 weeks). We stratified our analyses to evaluate possible effect modification by maternal age at conception (18-22, $23-26$, and $\geq 27$ years old), age at vaccination (18-22, $23-26$, and $\geq 27$ years old), and age at enrolment to the first phase of the trial (18-22, 23-26, and $\geq 27$ years old).

\section{Sensitivity analyses}

To be sure that we did not miss a clinically important effect in a subgroup, we performed a series of sensitivity analyses. To explore the impact of including the unvaccinated cohort in the unexposed pregnancies, we estimated the miscarriage risk after excluding pregnancies in the unvaccinated cohort. To evaluate our assumption that the miscarriage rate after hepatitis A vaccination is the same as in the unvaccinated cohort, we compared the rates in these two groups. To rule out an effect of hepatitis A vaccine, we excluded pregnancies in the hepatitis A vaccine group using the unvaccinated cohort as the reference group. To rule out an effect of the inoculation regardless of antigen, and to rule out an effect of hepatitis A vaccine, we also considered the miscarriage rate in pregnancies by the same time windows as for the bivalent HPV vaccine pregnancies $(<90$ days $v \geq 90$ days) in women who received hepatitis A vaccine but not bivalent HPV vaccine, and using the unvaccinated cohort as the reference group.

Finally, we examined the risk of miscarriage based on the number of doses (one, two, or three) of bivalent HPV vaccine that a woman received during the time window of less than 90 days between estimated conception and 
vaccination by counting the number of doses administered in less than 90 days before or after the estimated conception; and at any time before the end of pregnancy by counting the number of doses received before the date of pregnancy resolution.

\section{Statistical analysis}

We performed all analyses under the intention to treat principle. For the metric of the effect of bivalent HPV vaccination on miscarriage we used the relative risk and corresponding 95\% confidence intervals (see supplementary file). Based on these regressions we estimated the predicted probability and 95\% confidence intervals thereof of miscarriage for vaccinated and unvaccinated women taking random variation into account. Additionally, we used generalized estimating equations, which allow the pregnancy outcomes from the same woman to be unconditionally correlated; the two methods gave practically identical estimates and 95\% confidence intervals. The rates of live births and the ratios thereof were estimated in the same way. To estimate the effect of bivalent HPV vaccine on the total number of pregnancies, we computed the rates of total number of pregnancies using a denominator of person time (see supplementary file).

We performed analyses with and without adjustments for well known and suspected confounders. There are few well established risk factors for miscarriage, ${ }^{21}$ and even fewer that could confound the association between bivalent HPV vaccination and miscarriage. Covariates included age at conception (18-22, 23-26, 27-30, and >30 years old), calendar year of conception (two year intervals), and age at vaccination (18-22, 23-26, and $\geq 27$ years old). Maternal age at conception is unlikely to be a meaningful adjustment variable because the age of women during the long term follow-up is 22-29 years. We also did not adjust for history of pregnancy loss because an earlier and a subsequent pregnancy loss can share causal risk factors. ${ }^{22}$

For analyses involving bivalent HPV vaccination, reported $\mathrm{P}$ values are one sided because a protective effect of the vaccination against miscarriage does not constitute a safety concern; thus we considered associations to be statistically significant when one sided $P$ values were $<0.025$. $P$ values for estimates of miscarriage among unexposed pregnancies are two sided. Owing to the exploratory nature and the number of subgroup analyses, results should be interpreted with caution, as nominally significant associations may arise by chance alone. ${ }^{23}$ Analyses were done in SAS 9.2.

\section{False positive report probability}

We evaluated how a positive association between bivalent HPV vaccine and miscarriage risk might be true by estimating the false positive report probability. ${ }^{24}$ This method computes the probability of no true effect of bivalent HPV vaccine on miscarriage given a claim of positivity based on the $\mathrm{P}$ value, given the prior probability of a real association, and the statistical power to find an effect under a specified alternative hypothesis.
A detailed description of the method and its interpretation is provided in the supplementary file.

\section{Meta-analysis of CVT and parallel PATRICIA trial}

To update our previous report, ${ }^{9}$ we performed a meta-analysis of the estimates of miscarriage risk in the Costa Rica HPV Trial and parallel PApilloma TRIal against Cancer In young Adults (PATRICIA) trial. To avoid the use of overlapping data from the Costa Rica HPV Trial, we estimated the relative risk of miscarriage less than 90 days from bivalent HPV vaccination and overall for exposed and unexposed pregnancies, using data accrued after the original report. ${ }^{9}$ We synthesized this estimate with the published pooled miscarriage relative risk from PATRICIA and the Costa Rica HPV Trial ${ }^{9}$ using fixed effect meta-analysis with Mantel-Haenszel weights. Heterogeneity was assessed with Cochran's Q and was quantified with the $\mathrm{I}^{2}$ metric. ${ }^{25}$ Meta-analysis was done in STATA 13.

\section{Patient involvement}

No patients were involved in setting the research question or the outcome measures, nor were they involved in the design and implementation of the study. There are no plans to involve patients in dissemination.

\section{Results \\ Miscarriage}

A total of 6621 exposed $(n=3394)$ and unexposed $(n=3227)$ pregnancies from 4864 women were included in the analysis (fig 1). Table 1, supplementary table 1 , and supplementary fig 1 show the number of eligible pregnancies and corresponding miscarriages. A total of 3394 pregnancies were conceived at any time from bivalent HPV vaccination, with 451 ending in miscarriage (13.3\%, 95\% confidence interval $12.2 \%$ to $14.4 \%)$. Among those, 381 pregnancies were conceived less than 90 days from bivalent HPV vaccination, of which 50 ended in miscarriage (13.1\%, 95\% confidence interval $9.7 \%$ to $16.5 \%$ ). The distribution of conception dates in relation to the vaccination date for exposed pregnancies and miscarriages thereof is shown in supplementary table 2 . Overall, 3327 pregnancies were unexposed to bivalent HPV vaccination, of which 414 ended in miscarriage $(12.8 \%, 95 \%$ confidence interval $11.7 \%$ to $14.0 \%$ ).

\section{Bivalent HPV vaccination $<90$ days from conception} Vaccination with bivalent HPV less than 90 days from conception was not associated with an increased risk of miscarriage in either unadjusted analyses or when adjusting for age at conception, calendar year, and age at vaccination (table 2). The corresponding predicted risks were similar for vaccinated and unvaccinated women (see supplementary fig 2A). Similarly, no association between bivalent HPV vaccination and miscarriage risk was seen when we restricted the analysis to miscarriages that occurred at 0-6, 7-12, and 13-20 weeks of gestation, as well as in analyses stratified by age at conception, age at enrolment, and age at vaccination. Results were 


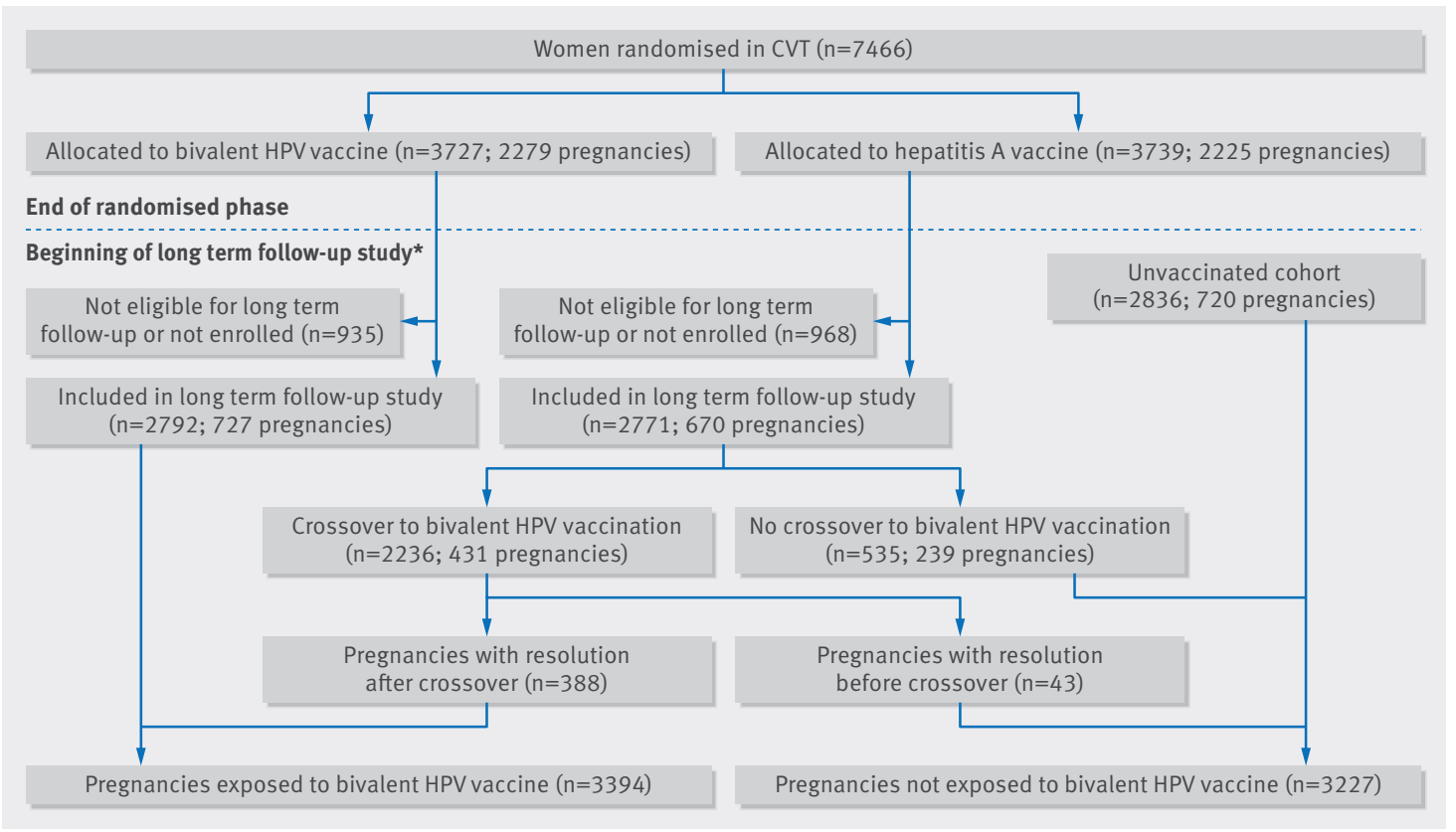

Fig 1 Exposed and unexposed pregnancies in Costa Rica HPV Vaccine Trial (CVT) and long term follow-up study. Shown are relative risks of miscarriage for pregnancies conceived any time since vaccination with bivalent human papillomavirus (HPV) vaccine in the previously published pooled analysis of CVT and parallel PApilloma TRIal against Cancer In young Adults (PATRICIA) ${ }^{9}$ and in update of CVT, including data acquired after our previous report ${ }^{9}$ as well as corresponding fixed effect summary relative risks. Data are shown overall and by gestational age of miscarriage. *Long term follow-up study was implemented for all women enrolled in trial (except those living in Puntarenas)

similar using generalized estimating equations with practically identical 95\% confidence intervals (see supplementary table 3$)$.

In subgroup analyses focusing on pregnancies conceived less than 90 days from both bivalent HPV and hepatitis A vaccinations, the miscarriage rate in pregnancies conceived less than 90 days from bivalent HPV vaccination (13.1\%) was slightly lower than in those conceived less than 90 days from hepatitis A vaccination (13.6\%), with no evidence of association between

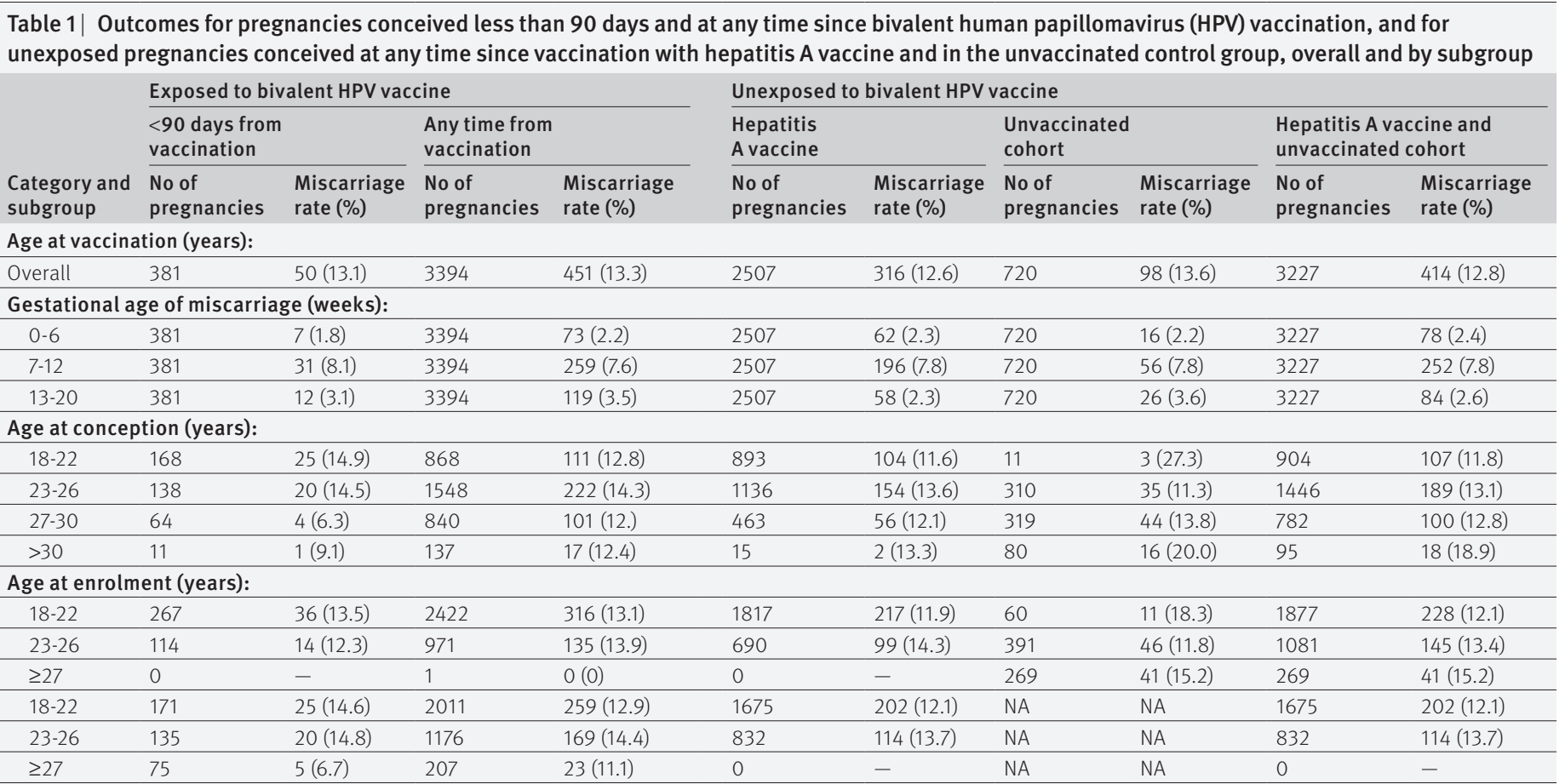

$\mathrm{NA}=$ not applicable because no vaccination was performed in the unvaccinated cohort; $\mathrm{NP}=$ not pertinent because the miscarriage rate in the bivalent HPV vaccine group was smaller than in the control group. 


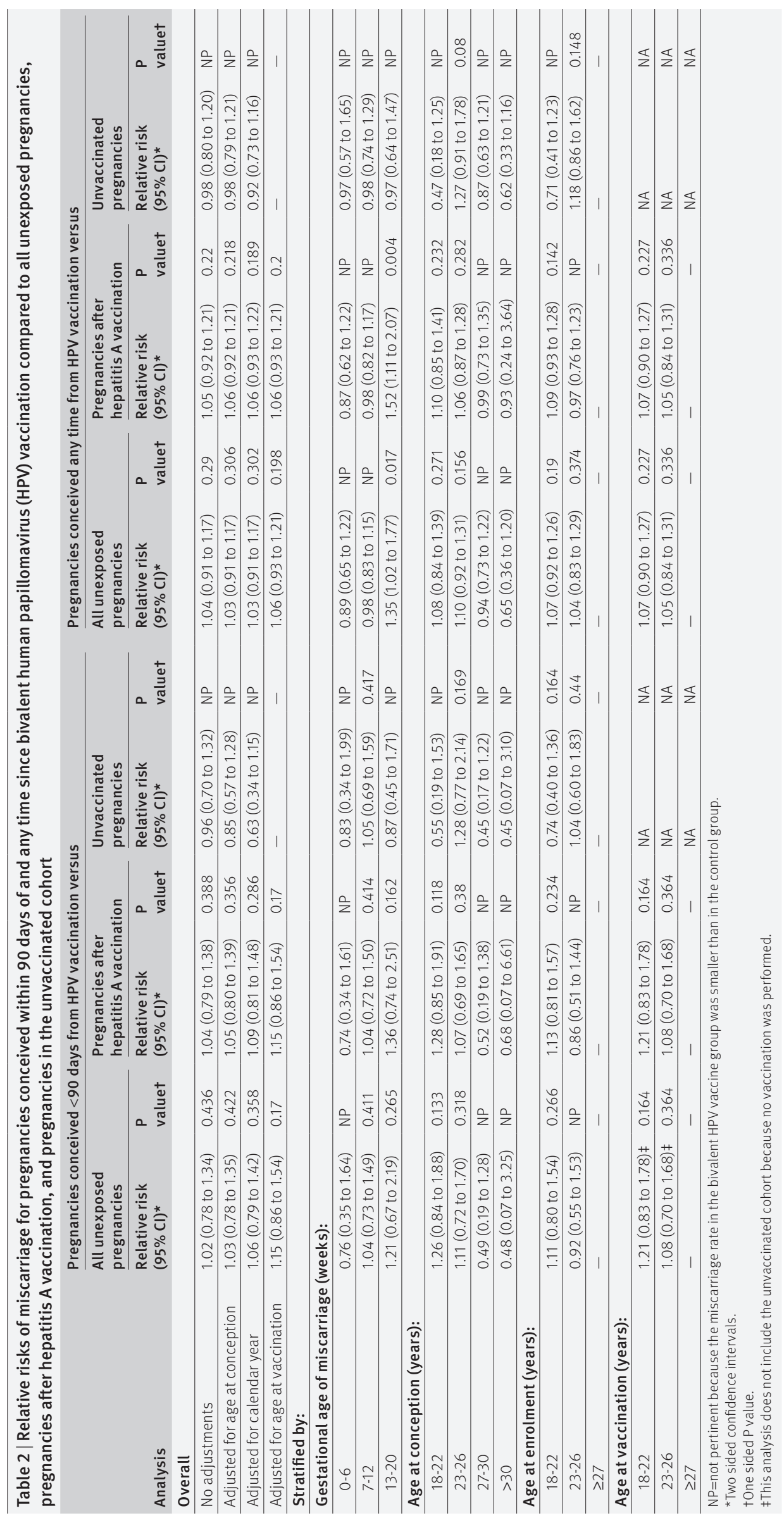

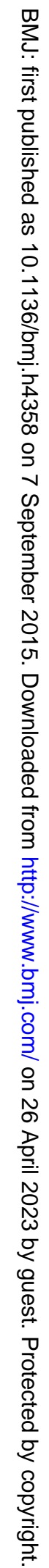


bivalent HPV vaccination and miscarriage in any of the analyses (see supplementary table 4).

Bivalent HPV vaccination any time from conception In both unadjusted and adjusted analyses there was no effect of bivalent HPV vaccination for pregnancies conceived any time from vaccination (table 2); results were the same when we stratified on age at conception, age at enrolment, and age at vaccination. The corresponding predicted risks were similar for vaccinated and unvaccinated women (see supplementary fig 2B). When we restricted our endpoint to the gestational week of miscarriage, bivalent HPV vaccination had no effect on risk of miscarriage in weeks $0-6$ or 7-12 of gestation. The risk of miscarriage was increased in weeks 13-20 of gestation (relative risk 1.35, 95\% confidence interval 1.02 to 1.77 , one sided $\mathrm{P}=0.017$ ). Results were similar using generalized estimating equations with practically identical 95\% confidence intervals (see supplementary table 5). Applying the method of false positive report probability, the value was consistently greater than 0.1 for any plausible prior probability and relative risk (see supplementary table 6), suggesting that the association may be an artifact of a thorough set of sensitivity and subgroup analyses, although a genuine association cannot be totally ruled out.

\section{Sensitivity analyses}

The results remained practically unchanged when excluding pregnancies in the unvaccinated cohort and pregnancies exposed to hepatitis A vaccine (table 2) from the unexposed pregnancies. The nominally significant association pertained to an increased risk of miscarriage at weeks 13-20 of gestation for pregnancies conceived any time since bivalent HPV vaccination compared with pregnancies exposed to hepatitis A vaccine (relative risk 1.52, 95\% confidence interval 1.11 to 2.07; one sided $\mathrm{P}=0.004)$. If we assume prior probabilities of 0.1 or more and relative risk of 2.00 or more in the false positive report probability analysis (see supplementary table 6), the effect of bivalent HPV vaccination on miscarriage becomes noteworthy (false positive report probability $<0.1$ ). Such a high relative risk may be thought unlikely however, as it would presumably have been detected in previous randomized and observational studies.

The miscarriage rate among pregnancies conceived any time since hepatitis A vaccination was slightly lower than the rate in the unvaccinated cohort (12.6\% $v 13.6 \%, \mathrm{P}=0.475$; see supplementary table 7 ). For pregnancies conceived less than 90 days from hepatitis A vaccination (see supplementary table 8 ), the risk of miscarriage was increased for ages 23-26 years at conception (relative risk 1.98, 95\% confidence interval 1.15 to 3.42, $\mathrm{P}=0.014$ ) and ages $23-26$ years at enrolment (relative risk 1.87, 95\% confidence interval 1.08 to 3.25, $\mathrm{P}=0.026)$. Similar results were obtained when comparing miscarriage rates for pregnancies conceived less than 90 days with those conceived 90 days or more after hepatitis A vaccination (see supplementary table 9).
There was no association between bivalent HPV vaccination and miscarriage for pregnancies exposed to one dose (see supplementary tables 10,12, and 15) or two doses (see supplementary tables 11, 13, and 16). With three doses of bivalent HPV vaccination before the pregnancy resolution, the risk of miscarriage was increased within a few subgroups: for pregnancies conceived less than 90 days from vaccination for women aged 18-22 years at enrolment (relative risk 1.63, 95\% confidence interval 1.07 to 2.48 , one sided $\mathrm{P}=0.012$; see supplementary table 14) and for pregnancies at weeks 13-20 of gestation regardless of the time between conception and vaccination (relative risk 1.38, 95\% confidence interval 1.03 to 1.85 , one sided $\mathrm{P}=0.014$; see supplementary table 17). As shown in supplementary table 6, the false positive report probability was greater than 0.1 for the first association under any plausible prior probability or relative risk, whereas a relative risk would have to be at least 1.50 and the prior probabilities 0.1 or more for the second association to be likely to be true.

\section{Meta-analysis with previous data}

An additional 5138 exposed and unexposed pregnancies occurred in the trial and post-trial phases (supplementary text) after the publication of our previous report. ${ }^{9}$ The unadjusted relative risk of miscarriage for pregnancies conceived less than 90 days from bivalent HPV vaccination was 0.62 (95\% confidence interval 0.35 to 1.11). After meta-analysis with the previously published pooled estimate (relative risk 1.49, 95\% confidence interval 1.04 to 2.15) from the parallel PATRICIA trial and Costa Rica HPV Vaccine Trial, ${ }^{9}$ the summary relative risk was 1.11 (95\% confidence interval 0.82 to 1.51 ; one sided $\mathrm{P}=0.243 ; \mathrm{I}^{2}=84.5 \%, \mathrm{P}=0.011$ for heterogeneity). For pregnancies conceived any time since vaccination with bivalent HPV (fig 2), there was no risk of miscarriage overall or by gestational age except for miscarriages in weeks 13-20 of gestation (relative risk 1.34, $95 \%$ confidence interval 1.03 to 1.73 , one sided $\mathrm{P}=0.013$ ).

\section{Live births and total number of pregnancies}

There was no association between bivalent HPV vaccination and rates of live births (table 3; see supplementary table 18) or rates of total pregnancies (relative risk $0.98,95 \%$ confidence interval 0.93 to 1.03; one sided $\mathrm{P}=0.177)$. See supplementary file for further information.

\section{Discussion}

A previous analysis of miscarriage risk in two large randomized controlled clinical trials of human papillomavirus (HPV) vaccine found no evidence of an increased risk among pregnancies conceived at least 90 days after vaccination. ${ }^{9}$ That previous study, however, could not exclude the possibility that pregnancies conceived closer to the time of vaccination might be at increased risk. ${ }^{9}$ We have used new data from the extended follow-up of those clinical trial cohorts to explore this possible risk. The updated evidence provided here shows that vaccination with bivalent vaccine against 


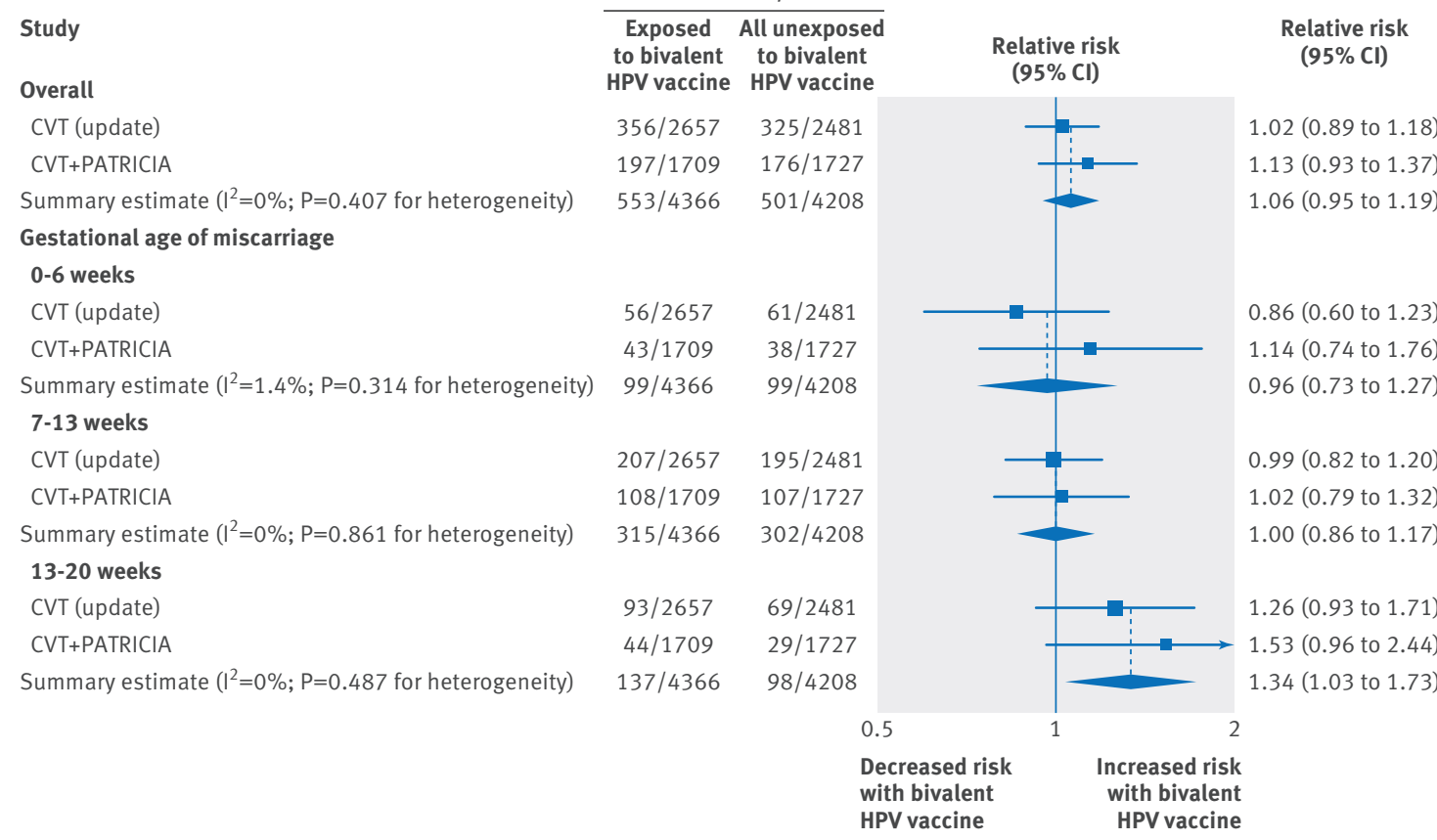

Fig 2 Meta-analysis on risk of miscarriage for pregnancies conceived any time since vaccination with bivalent HPV overall and by gestational age in Costa Rica HPV Trial (CVT) and parallel PATRICIA trial

HPV types 16 and 18 is not associated with miscarriage for pregnancies conceived less than 90 days from vaccination. Data from more than 6000 pregnancies from the randomized and post-trial phases of the Costa Rica HPV Vaccine Trial and a population based unvaccinated cohort do not support an increased risk of miscarriage in pregnancies conceived in this time window. Sensitivity, subgroup, and stratified analyses showed no effect of potential sources of systematic errors or subgroup specific effects (inclusion of the unvaccinated cohort, number of doses, age at conception, age at enrolment, gestational age of miscarriage). The present analysis did find an increased risk of miscarriage among one subgroup of pregnancies conceived at any time after vaccination-namely, 13-20 weeks of gestation. While this finding had not been suggested in the previous data, the result remained after meta-analysis of the new data with previously published results from the PATRICIA trial. A small increase in risk for miscarriages in weeks 13-20 of gestation cannot totally be ruled out, though it is also compatible with chance.

\section{Comparison with other studies}

Two pooled analyses by the vaccine manufacturer did not find a difference in miscarriage risk for pregnancies conceived in a risk window of -30 to 45 days from bivalent HPV vaccination. ${ }^{2627}$ Similarly, a lack of association was observed in placebo controlled trials evaluating the efficacy and safety of the bivalent HPV vaccine. ${ }^{2829}$ Nevertheless, a subgroup analysis in black women in the Females United to Unilaterally Reduce Endo/Ectocervical Disease (FUTURE) I/II trials showed an increased risk of miscarriage compared with unvaccinated pregnancies for the quadrivalent HPV vaccine that has a different adjuvant (amorphous aluminum hydroxyphosphate sulfate). ${ }^{30}$ However, no results were reported for restricting the endpoint by different periods of gestation as in our study, which did not confirm the overall increased miscarriage risk in FUTURE I/II using longer follow-up and a larger number of pregnancies.

A systematic review and evidence synthesis on vaccine safety by the US Agency for Health Research and Quality found insufficient evidence for causality between HPV vaccination and miscarriage risk. ${ }^{620}$ This review updated a previous report by the Institute of Medicine, which also concluded that the evidence for a causal relation between HPV vaccination and any major adverse event is inadequate. ${ }^{7}$ Our analyses investigating this association in an independent dataset not included in the previous reports further confirm that an effect of the HPV bivalent vaccine on miscarriage is highly unlikely. The evidence in the Agency for Health Research and Quality and Institute of Medicine reports was based on trials that used unvaccinated control groups (that is, women who did not receive any type of control vaccination), excluding studies that used hepatitis A vaccine or other vaccines as control interventions. The conclusions of these reports are in agreement with our sensitivity analyses comparing the miscarriage rates in pregnancies exposed to bivalent HPV vaccination with pregnancies in the unvaccinated cohort, which found no difference in the miscarriage rates between the two groups.

\section{Possible explanations and implications for clinicians and policy makers}

Miscarriage is a common outcome of pregnancy, occurring in $10-15 \%$ of all clinically recognized pregnancies. Given the precautions taken to avoid vaccination of 


\begin{tabular}{|c|c|c|c|c|c|c|c|c|c|c|}
\hline \multirow[b]{2}{*}{ Analysis } & \multicolumn{2}{|c|}{ All unexposed pregnancies } & \multicolumn{4}{|c|}{ Pregnancies conceived $<90$ days from HPV vaccination } & \multicolumn{4}{|c|}{ Pregnancies conceived any time after HPV vaccination } \\
\hline & $\begin{array}{l}\text { No of } \\
\text { pregnancies }\end{array}$ & $\begin{array}{l}\text { No of live } \\
\text { births (\%) }\end{array}$ & $\begin{array}{l}\text { No of } \\
\text { pregnancies }\end{array}$ & $\begin{array}{l}\text { No of live } \\
\text { births (\%) }\end{array}$ & $\begin{array}{l}\text { Relative risk } \\
(95 \% \mathrm{Cl})\end{array}$ & $\begin{array}{l}P \\
\text { value }\end{array}$ & $\begin{array}{l}\text { No of } \\
\text { pregnancies }\end{array}$ & $\begin{array}{l}\text { No of live } \\
\text { births (\%) }\end{array}$ & $\begin{array}{l}\text { Relative risk } \\
(95 \% \mathrm{CI})\end{array}$ & $\begin{array}{l}P \\
\text { value }\end{array}$ \\
\hline \multicolumn{11}{|l|}{ Overall } \\
\hline No adjustments & 3188 & 2739 (85.9) & 380 & $325(85.5)$ & $1.00(0.95$ to 1.04$)$ & 0.838 & 3353 & $2872(85.7)$ & $1.00(0.98$ to 1.02$)$ & 0.762 \\
\hline $\begin{array}{l}\text { Adjusted for age } \\
\text { at conception }\end{array}$ & 3188 & 2739 (85.9) & 380 & $325(85.5)$ & 0.99 (0.95 to 1.04$)$ & 0.742 & 3353 & $2872(85.7)$ & 1.00 (0.98 to 1.02) & 0.782 \\
\hline $\begin{array}{l}\text { Adjusted for } \\
\text { calendar year }\end{array}$ & 3188 & $2739(85.9)$ & 380 & $325(85.5)$ & 0.99 (0.95 to 1.04) & 0.728 & 3353 & $2872(85.7)$ & 1.00 (0.98 to 1.02) & 0.828 \\
\hline $\begin{array}{l}\text { Adjusted for age } \\
\text { at vaccination* }\end{array}$ & 3188 & 2739 (85.9) & 380 & $325(85.5)$ & 0.98 (0.93 to 1.03) & 0.34 & 3353 & $2872(85.7)$ & 0.99 (0.97 to 1.01) & 0.503 \\
\hline \multicolumn{11}{|l|}{ Stratified by: } \\
\hline \multicolumn{11}{|c|}{ Age at conception (years): } \\
\hline $18-22$ & 903 & $786(87.0)$ & 168 & $141(83.9)$ & $0.96(0.90$ to 1.04$)$ & 0.313 & 867 & $750(86.5)$ & 0.99 (0.96 to 1.03$)$ & 0.739 \\
\hline $23-26$ & 1437 & $1233(85.8)$ & 137 & $115(83.9)$ & 0.98 (0.91 to 1.06) & 0.573 & 1540 & $1304(84.7)$ & 0.99 (0.96 to 1.02) & 0.385 \\
\hline $27-30$ & 764 & $655(85.7)$ & 64 & $59(92.2)$ & $1.08(1.00$ to 1.16$)$ & 0.065 & 816 & $705(86.4)$ & 1.01 (0.97 to 1.05) & 0.703 \\
\hline$>30$ & 84 & $65(77.4)$ & 11 & $10(90.9)$ & $1.17(0.94$ to 1.46$)$ & 0.151 & 129 & $112(86.8)$ & $1.12(0.98$ to 1.28$)$ & 0.092 \\
\hline \multicolumn{11}{|c|}{ Age at enrolment (years): } \\
\hline $18-22$ & 1869 & $1619(86.6)$ & 266 & $227(85.3)$ & $0.99(0.93$ to 1.04$)$ & 0.58 & 2391 & $2057(86)$ & 0.99 (0.97 to 1.02) & 0.576 \\
\hline $23-26$ & 1063 & $908(85.4)$ & 114 & $98(86.0)$ & 1.01 (0.93 to 1.09) & 0.873 & 961 & $814(84.7)$ & 0.99 (0.96 to 1.03$)$ & 0.652 \\
\hline$\geq 27$ & 256 & $212(82.8)$ & 0 & - & - & - & 1 & $1(100.0)$ & $1.20(1.20$ to 1.20$)$ & - \\
\hline \multicolumn{11}{|c|}{ Age at vaccination (years)*: } \\
\hline $18-22$ & 1669 & $1446(86.6)$ & 171 & $144(84.2)$ & 0.97 (0.91 to 1.04) & 0.41 & 1991 & $1716(86.2)$ & 0.99 (0.97 to 1.02) & 0.692 \\
\hline $23-26$ & 830 & $708(85.3)$ & 134 & $112(83.6)$ & $0.98(0.90$ to 1.06$)$ & 0.619 & 1160 & $978(84.3)$ & 0.99 (0.95 to 1.03) & 0.543 \\
\hline$\geq 27$ & 0 & - & 75 & $69(92.0)$ & - & - & 202 & $178(88.1)$ & - & - \\
\hline
\end{tabular}

pregnant women, these large clinical trials generated a relatively limited number of pregnancies for analysisespecially when searching within possibly susceptible subgroups of pregnancies or women. The few positive associations between bivalent HPV vaccination and miscarriage risk found among the large number of sensitivity and subgroup analyses should be interpreted with caution. There is no established pathophysiological mechanism through which bivalent HPV vaccination would affect the risk of miscarriage. A theoretical argument involves alterations in the maternal immune system during early pregnancy ${ }^{31}$ caused by the ASO4 adjuvant, which consists of aluminum phosphate and monophosphoryl lipid A. However, other ASO4 based vaccines have not been associated with miscarriage risk. ${ }^{32}$ More generally, evidence for a causal role of autoimmunity in itself on miscarriage risk is weak. ${ }^{21}$ Meanwhile, the vaccine has not been associated with autoimmune conditions (such as antiphospholipid syndrome and thyroid autoimmunity) that have been linked to miscarriage.

The positive associations could reflect differences in the detection of early pregnancy among exposed and unexposed women, as at the time of crossover vaccination women were not required to undergo a pregnancy test before hepatitis A vaccination as in the blinded phase of the trial. This may have resulted in underdetection-or detection only after the first trimester-of pregnancies and therefore under-detection of miscarriages in the unexposed group, thus leading to an artificial increase in miscarriage risk. Also, the relative risk changed direction $(<1)$ when we compared the risks of miscarriage in pregnancies exposed to bivalent HPV vaccination with those in the unvaccinated cohort.
According to the false positive report probability analysis, the association is noteworthy only when the risk among pregnancies exposed to bivalent HPV vaccine is compared with risk among pregnancies exposed to hepatitis A vaccine.

Safety surveillance data from national immunization programs have suggested no difference in the rates of miscarriage between pregnancies in women vaccinated with bivalent HPV and the unvaccinated population. ${ }^{33}$ That result is consistent with our findings that the miscarriage rates for exposed pregnancies and pregnancies in the unvaccinated cohort did not differ statistically significantly. Possible explanations of the increased miscarriage risk in pregnancies exposed to bivalent HPV vaccine compared with those exposed to hepatitis A vaccine include a reduced risk of miscarriage among women who received hepatitis A vaccine as suggested before ${ }^{27}$ or by chance. Indeed, the miscarriage rate in weeks 13-20 in pregnancies exposed to hepatitis A vaccine was not only lower than the risk among the pregnancies exposed to bivalent HPV vaccination but also lower than among the unvaccinated cohort.

Policy makers have issued reassuring reports on the safety of the two HPV vaccines, both overall and for pregnancy related outcomes specifically. The most recent evidence reviews used in recommendations from the Advisory Committee on Immunization Practices ${ }^{2}$ and $\mathrm{WHO}^{8}$ conclude that the public health benefits of HPV vaccination outweigh potential harms. Our analyses, which do not indicate an increased miscarriage risk either overall or for pregnancies conceived less than 90 days after vaccination, provide additional support to these recommendations. Only about 3\% of pregnancies end in miscarriage after the 12th week of gestation. ${ }^{3134}$ 
Thus the absolute risk of miscarriage associated with bivalent HPV vaccination in weeks 13-20 after gestation is small (in our study, the absolute difference in miscarriage rates was $0.9 \%$ (that is, 3.5\% minus 2.6\%), table 1 ). In the event that such a small risk difference was to be established as real, even then it would have to be weighed against the well documented public health benefits of vaccination in preventing precancerous cervical lesions. ${ }^{35}$

\section{Limitations of this study}

Our study has some limitations that should be acknowledged. Firstly, there was differential determination of pregnancy before hepatitis A vaccination in the blinded phase compared with the long term follow-up study, as women who received crossover vaccination were not required to undergo a pregnancy test. This could result in fewer undetected pregnancies exposed to hepatitis A vaccine, thereby explaining the slightly increased risk of miscarriage after vaccination with bivalent HPV as well as the differences observed when excluding pregnancies exposed to hepatitis A vaccine from the unexposed pregnancies. Secondly, women never vaccinated with bivalent HPV were followed-up less frequently after the blinded trial phase than women who were vaccinated with bivalent HPV. This differential follow-up might increase the ascertainment of events in the pregnancies exposed to bivalent HPV vaccine, thereby overestimating the risk of miscarriage in this group. Thirdly, the post-trial crossover vaccination with either bivalent HPV or hepatitis A may have been influenced by many factors and could be subject to unmeasured confounding, as women were no longer randomized. Results, though, remained largely unchanged when we adjusted for potential confounders. Fourthly, there are limitations of false positive report probability analyses. ${ }^{2436}$ As with all bayesian methods, the false positive report probability approach incorporates subjectivity, particularly in assigning values for prior probabilities, and the final values are not equivalent to bayesian posterior probabilities. Notwithstanding these limitations, false positive report probability has the advantage of formally combining "hard" evidence from studies with explicit (even if subjective) information. Finally, we considered pregnancies in the hepatitis A vaccine arm and those in the unvaccinated cohort, which was ascertained outside the trial setting, but combining the two groups did not affect our inferences, as shown by sensitivity analyses, which was anticipated as the two groups are comparable. ${ }^{15}$

\section{Conclusions and implications for future research}

We found no evidence to support the single questionable positive finding of an increased risk of miscarriage from bivalent HPV vaccination reported previously for pregnancies conceived less than 90 days after vaccination. ${ }^{9}$ The increased risk of miscarriage in one subgroup might be expected, given the thoroughness of our evaluation. Though there is no biological or pathophysiological mechanism to explain a potential association between HPV vaccination and miscarriage, it would be possible to explore this question further through systematic evaluations and synthesis of existing published and unpublished literature-in particular the clinical study reports from the vaccine manufacturers ${ }^{37} 38$ and independent reanalysis of trial data. ${ }^{3940}$ Towards this end, recent data sharing policies by regulatory agencies in Europe ${ }^{41}$ and the United States ${ }^{42}$ could facilitate such efforts. ${ }^{43}$ Most trials on the bivalent and quadrivalent HPV vaccines have been conducted on adolescents and young adults, and hence the long term safety of the vaccines in 9-11 year old girls who are routinely vaccinated has not been extensively evaluated in randomized trials. Although no pregnancy related safety issues have been recognized to date, ${ }^{44}$ and the comparative safety of most interventions for children and adult populations is on average the same, ${ }^{45}$ monitoring of the currently available immunization programs (through the Vaccine Adverse Event Reporting System, the Vaccine Safety Datalink, and the Vaccines and Medications in Pregnancy Surveillance System) could further establish the safety of the currently available HPV vaccines.

Available data support the safe continuation of HPV vaccination programs according to current guidelines. As with other vaccines, ${ }^{6}$ any concerns about possible adverse events must be weighed against the established benefits of vaccination for individuals and the community.

Investigators in the Costa Rica HPV Vaccine Trial (CVT) Group Proyecto Epidemiológico Guanacaste, Fundación INCIENSA, San José, Costa Rica-Bernal Cortés (specimen and repository manager), Paula González (long term follow-up study: co-principal investigator), Rolando Herrero (CVT: co-principal investigator), Silvia E Jiménez (trial coordinator), Carolina Porras (co-investigator), and Ana Cecilia Rodríguez (co-investigator). United States National Cancer Institute (NCI), Bethesda, MD, USA-Allan Hildesheim (co-principal investigator and $\mathrm{NCl}$ co-project officer), Aimée R Kreimer (long term follow-up study: co-principal investigator and $\mathrm{NCl}$ co-project officer), Douglas R Lowy (human papillomavirus (HPV) virologist), Mark Schiffman (CVT: medical monitor and NCl co-project officer), John T Schiller (HPV virologist), Mark Sherman (CVT: quality control pathologist), and Sholom Wacholder (statistician). Leidos Biomedical Research, Frederick National Laboratory for Cancer Research, Frederick, MD, USA (HPV Immunology Laboratory)—Ligia A Pinto and Troy J Kemp. Georgetown University, Washington, DC, USA-Mary K Sidawy (CVT: histopathologist). DDL Diagnostic Laboratory, Netherlands (HPV DNA testing)-Wim Quint, Leen-Jan van Doorn, and Linda Struijk. University of California, San Francisco, CA, USA-Joel M Palefsky and Teresa M Darragh. University of Virginia, Charlottesville, VA, USA-Mark H Stoler.

We thank the women of Guanacaste and Puntarenas, Costa Rica, fo their participation. In Costa Rica, we acknowledge the effort and dedication of the staff involved in this project; we would like to specifically acknowledge the contributions by Loreto Carvajal, Rebeca Ocampo, Cristian Montero, Diego Guillen, Jorge Morales, and Mario Alfaro. In the United States, we thank the team from Information Management Services responsible for the development and maintenance of the data system used in the trial and who serve as the data management center for this effort, especially Jean Cyr, Julie Buckland, John Schussler, and Brian Befano. We thank Diane Solomon (CVT: medical monitor and QC pathologist) for her invaluable contributions during the randomized blinded phase of the trial and the design of the long term follow-up study, and Nora Macklin (CVT) and Kate Torres (long term follow-up study) for the expertise in coordinating the study. We thank the members of the data and safety monitoring board charged with protecting the safety and interest of participants during the randomized, blinded phase of our study (Steve Self, chair, Adriana Benavides, Luis Diego Calzada, Ruth Karron, Ritu Nayar, and Nancy Roach) and members of the external Scientific HPV Working Group who have contributed to the success of our efforts over the years (Joanna Cain, chair, Diane Davey, David DeMets, Francisco Fuster, Ann Gershon, Elizabeth Holly, Silvia Lara, Henriette Raventós, 
Wasima Rida, Luis Rosero-Bixby, Kristen Suthers, Amber D’Souza, Richard Roden, and Peter Gilbert).

Contributors: OAP, AJW, and SW designed the current analysis. OAP, BLB, AJW, and SW performed the statistical analysis. PG, ACR, RH, JTS, ARK, MS, and AH collected the data. OAP, MS, AH, AJW, and SW interpreted the data; OAP wrote the first draft of the manuscript with input from AJW and SW. BLB, PG, ACR, RH, JTS, ARK, MS, and $A H$ reviewed the first draft of the manuscript and critically commented on it; all authors approved the final version of the manuscript. SW is the guarantor. All authors, external and internal, had full access to all of the data (including statistical reports and tables) in the study and can take responsibility for the integrity of the data and the accuracy of the data analysis. All members of the CVT Group designed the randomized trial and the long term follow-up study.

Funding: The Costa Rica HPV Vaccine Trial is a longstanding collaboration between investigators in Costa Rica and the US National Cancer Institute ( $\mathrm{NCl}$ ). The trial is sponsored and funded by the $\mathrm{NCl}$ (contract N01-CP-11005), with funding support from the National Institutes of Health Office of Research on Women's Health. GlaxoSmithKline (GSK) Biologicals provided vaccine and support for aspects of the trial associated with regulatory submission needs of the company under a clinical trials agreement (FDA BB-IND 7920) during the four year, randomized blinded phase of our study. This manuscript was not prepared in collaboration with GSK Biologicals investigators, and GSK Biologicals had no role in the study design and the collection, analysis, and interpretation of data from CVT in this paper, or in the writing of the article. GSK Biologicals took no part in the decision to submit an article for publication. The analysis reported here was supported by the intramural research programs of the $\mathrm{NCl}$ and the National Institute of Environmental Health Sciences, both parts of the National Institutes of Health.

Competing interests: All authors have completed the ICMJE uniform disclosure form at http://www.icmje.org/coi_disclosure.pd (available on request from the corresponding authors). JTS and DRL are named inventor on US government owned HPV vaccine patents that are licensed to GlaxoSmithKline and Merck and for which the National Cancer Institute $(\mathrm{NCl})$ receives licensing fees. They are entitled to limited royalties as specified by federal law. The other authors and investigators declare that they have no conflicts of interest. The researchers are completely independent from the non-government funders and sponsors. The $\mathrm{NCl}$, National Institute of Environmental Health Sciences, and Costa Rica investigators are responsible for the design and conduct of the study; collection, management, analysis, and interpretation of the data; and preparation of the manuscript. The findings and conclusions in this report are those of the authors and do not necessarily represent the official views of the US Department of Health and Human Services, the National Institutes of Health, the $\mathrm{NCl}$, the National Institute of Environmental Health Sciences, or any other funding agency. BLB is employed by a contractor company (Information Management Services) to the $\mathrm{NCl}$; nevertheless, there is no conflict of interest in the authors' submission, nor does this alter adherence to BMJ policies on sharing data.

Ethical approval: This study was approved by the relevant local or national research ethics committees. The CVT trial was approved by the institutional review boards of the US National Cancer Institute $(\mathrm{NCl})$, and the Instituto Costarricense de Investigación y Enseñanza en Nutrición y Salud (INCIENSA) in Costa Rica. The long term follow-up study was approved by the institutional review boards of the $\mathrm{NCl}$ and of the National University in Costa Rica. Participants gave informed consent before taking part in the studies. The

Corresponding author has the right to grant on behalf of all authors and does grant on behalf of all authors, an exclusive licence (or non exclusive for government employees) on a worldwide basis to the BMJ Publishing Group Ltd and its licensees, to permit this article to be published in BMJ editions and any other BMJPG products and to exploit all subsidiary rights, as set out in our licence (http://resources. bmj.com/bmj/authors/checklists-forms/licence-for-publication).

Data sharing: The full analytical dataset, technical appendix, and statistical code are available on request from ARK, the study co-principal investigator, at kreimera@mail.nih.gov. Participants' consent for data sharing was not obtained, but the presented data are anonymised and there is no risk of identification.

Transparency: The manuscript's guarantor (SW) affirms that the manuscript is an honest, accurate, and transparent account of the study being reported; that no important aspects of the study have been omitted; and that any discrepancies from the study as planned (and, if relevant, registered) have been explained.
This is an Open Access article distributed in accordance with the Creative Commons Attribution Non Commercial (CC BY-NC 4.0) license, which permits others to distribute, remix, adapt, build upon this work non-commercially, and license their derivative works on different terms, provided the original work is properly cited and the use is non-commercial. See: http://creativecommons.org/licenses/ by-nc/4.0/.

1 Centers for Disease Control and Prevention. FDA licensure of bivalent human papillomavirus vaccine (HPV2, Cervarix) for use in females and updated HPV vaccination recommendations from the Advisory Committee on Immunization Practices (ACIP). MMWR Morb Mortal Wkly Rep 2010;59:626-9.

2 Markowitz LE, Dunne EF, Saraiya M, et al. Human papillomavirus vaccination: recommendations of the Advisory Committee on Immunization Practices (ACIP). MMWR Morb Mortal Wkly Rep 2014;63:1-30

3 Kuehn BM. Immunization recommendations expanded for hepatitis B, HPV, pertussis vaccines. JAMA 2012;307:1353-4

4 Noronha AS, Markowitz LE, Dunne EF. Systematic review of human papillomavirus vaccine coadministration. Vaccine 2014:32·2670-4

5 Schiller JT, Castellsague X, Garland SM. A review of clinical trials of human papillomavirus prophylactic vaccines. Vaccine 2012;30(Suppl 5):F123-38.

6 Maglione MA, Gidengil C, Das L, et al. Safety of vaccines used for routine immunization in the United States. Evidence report/technology assessment No 215. Agency for Healthcare Research and Quality, 2014.

Institute of Medicine, Committee to Review Adverse Effects of Vaccines. In: Stratton K, Ford A, Rusch E, et al, eds. Adverse effects of vaccines: evidence and causality. IOM, 2011

8 Global Advisory Committee on Vaccine Safety. 11-12 December 2013. Wkly Epidemiol Rec 2014:89:53-60.

9 Wacholder S, Chen BE, Wilcox A, et al. Risk of miscarriage with bivalent vaccine against human papillomavirus (HPV) types 16 and 18: pooled analysis of two randomised controlled trials. BM/ 2010;340:c712.

10 Westra TA, Rozenbaum MH, Rogoza RM, et al. Until which age should women be vaccinated against HPV infection? Recommendation based on cost-effectiveness analyses. J Infect Dis 2011;204:377-84.

11 Canfell K. Monitoring HPV vaccination programmes. BM 2010;340:c1666.

12 Schuchat A. HPV “coverage." N Engl J Med 2015;372:775-6.

13 Herrero R, Hildesheim A, Rodriguez AC, et al. Rationale and design of a community-based double-blind randomized clinical trial of an HPV 16 and 18 vaccine in Guanacaste, Costa Rica. Vaccine 2008;26:4795-808.

14 Hildesheim A, Wacholder S, Catteau G, et al. Efficacy of the HPV-16/18 vaccine: final according to protocol results from the blinded phase of the randomized Costa Rica HPV-16/18 vaccine trial. Vaccine 2014;32:5087-97.

15 Gonzalez P, Hildesheim A, Herrero R, et al. Rationale and design of a long term follow-up study of women who did and did not receive HPV 16/18 vaccination in Guanacaste, Costa Rica. Vaccine 2015;33:2141-51.

16 Barfield WD, Committee on Fetus and Newborn. Standard terminology for fetal, infant, and perinatal deaths. Pediatrics 2011;128:177-81.

17 National Center for Health Statistics (US). State definitions and reporting requirements for live births, fetal deaths, and induced termination of pregnancy. 1997 revised ed. Department of Health and Human Services, Centers for Disease Control and Prevention, National Center for Health Statistics, 1997.

18 Moro PL, Museru OI, Niu M, et al. Reports to the Vaccine Adverse Event Reporting System after hepatitis A and hepatitis AB vaccines in pregnant women. Am J Obstet Gynecol 2014;210:561 e1-6.

19 Irving GJ, Holden J, Yang R, et al. Hepatitis A immunisation in persons not previously exposed to hepatitis A. Cochrane Database Syst Rev 2012;7:CD009051.

20 Maglione MA, Das L, Raaen L, et al. Safety of vaccines used for routine immunization of US children: a systematic review. Pediatrics 2014; 134:325-37.

21 Larsen EC, Christiansen OB, Kolte AM, et al. New insights into mechanisms behind miscarriage. BMC Med 2013:11:154

22 Rai R, Regan L. Recurrent miscarriage. Lancet 2006;368:601-11.

23 Lagakos SW. The challenge of subgroup analyses-reporting without distorting. N Engl J Med 2006;354:1667-9.

24 Wacholder S, Chanock S, Garcia-Closas M, et al. Assessing the probability that a positive report is false: an approach for molecular epidemiology studies. J Natl Cancer Inst 2004:96:434-42.

25 Higgins JP, Thompson SG, Deeks J], et al. Measuring inconsistency in meta-analyses. BMJ 2003;327:557-60

26 Descamps D, Hardt K, Spiessens B, et al. Safety of human papillomavirus (HPV)-16/18 AS04-adjuvanted vaccine for cervical cancer prevention: a pooled analysis of 11 clinical trials. Hum Vaccin 2009;5:332-40

27 Angelo MG, David MP, Zima J, et al. Pooled analysis of large and long-term safety data from the human papillomavirus-16/18-AS04adjuvanted vaccine clinical trial programme. Pharmacoepidemiol Drug Saf 2014;23:466-79. 
28 Harper DM, Franco EL, Wheeler CM, et al. Sustained efficacy up to 4.5 years of a bivalent L1 virus-like particle vaccine against human papillomavirus types 16 and 18: follow-up from a randomised control trial. Lancet 2006;367:1247-55.

29 Skinner SR, Szarewski A, Romanowski B, et al. Efficacy, safety, and immunogenicity of the human papillomavirus 16/18 AS04adjuvanted vaccine in women older than 25 years: 4-year interim follow-up of the phase 3, double-blind, randomised controlled VIVIANE study. Lancet 2014;384:2213-27.

30 Clark LR, Myers ER, Huh W, et al. Clinical trial experience with prophylactic human papillomavirus $6 / 11 / 16 / 18$ vaccine in young black women. J Adolesc Health 2013;52:322-9.

31 Goldhaber MK, Fireman BH. The fetal life table revisited: spontaneous abortion rates in three Kaiser Permanente cohorts. Epidemiology 1991;2:33-9.

32 Tavares F, Cheuvart B, Heineman T, et al. Meta-analysis of pregnancy outcomes in pooled randomized trials on a prophylactic adjuvanted glycoprotein D subunit herpes simplex virus vaccine. Vaccine 2013;31:1759-64.

33 Angelo MG, Zima J, Tavares Da Silva F, et al. Post-licensure safety surveillance for human papillomavirus-16/18-AS04-adjuvanted vaccine: more than 4 years of experience. Pharmacoepidemiol Drug Saf 2014;23:456-65.

34 Regan L, Braude PR, Trembath PL. Influence of past reproductive performance on risk of spontaneous abortion. $B M$ J 1989;299:541-5.

35 Kreimer AR, Struyf F, Del Rosario-Raymundo MR, et al. Efficacy of fewer than three doses of an HPV-16/18 AS04-adjuvanted vaccine: combined analysis of data from the Costa Rica Vaccine and PATRICIA trials. Lancet Oncol 2015;16:775-86.
36 Lucke JF. A critique of the false-positive report probability. Genet Epidemiol 2009;33:145-50

37 Doshi P. Jefferson T, Del Mar C. The imperative to share clinical study reports: recommendations from the Tamiflu experience. PLoS Med 2012;9:e1001201.

38 Kmietowicz Z. Clinical study reports are not commercially confidential, says UK government. BMJ 2013;347:f6942

39 Krumholz HM, Peterson ED. Open access to clinical trials data. JAMA 2014;312:1002-3.

40 Christakis DA, Zimmerman FJ. Rethinking reanalysis. JAMA 2013;310:2499-500

41 Eichler HG, Abadie E, Breckenridge A, et al. Open clinical trial data for all? A view from regulators. PLoS Med 2012;9:e1001202.

42 Hudson KL, Collins FS. Sharing and reporting the results of clinical trials. JAMA 2015;313:355-6.

43 Institute of Medicine. Sharing clinical trial data: maximizing benefits, minimizing risk. IOM, 2015.

44 Ferris D, Samakoses R, Block SL, et al. Long-term study of a quadrivalent human papillomavirus vaccine. Pediatrics 2014;134:e657-65.

45 Lathyris D, Panagiotou OA, Baltogianni M, et al. Safety of medical interventions in children versus adults. Pediatrics 2014;133:e666-73.

Web appendix: supplementary material (trial inclusion/exclusion criteria; trial design; sources of exposed and unexposed pregnancies; statistical methods; supplementary tables and figures) 